\title{
Entrenamiento y Adaptación Muscular. Sustratos y Vías Metabólicas para la Producción de Energía
}

\author{
MV., PhD. Federico Martín Boffi
}

Director del Laboratorio de Fisiología y Fisiopatología del Equino Deportivo, Departamento de Clínicas, Facultad de Ciencias Veterinarias, Universidad Nacional de La Plata, Buenos Aires, Argentina.

\begin{abstract}
RESUMO
Tres isoformas de MyHC han sido caracterizadas en músculo esquelético de equinos adultos a nivel proteico: tipo I, IIA y IIX. La distribución de estas MyHCs definen tres tipos puros de fibras que contienen una sola isoforma (tipo I, IIA y IIX) y dos tipos de fibras híbridas que expresan dos isoformas (I+IIA y IIAX). Las fibras híbridas IIAX existen en los músculos locomotores como una población estable y significativa. Dependiendo de la naturaleza del estímulo, la respuesta adaptativa puede tomar diferentes formas: hipertrofia, remodelación sin hipertrofia y respuesta mixta, cuando se combina la remodelación con la hipertrofia. Los equinos pueden aprovechar diferentes sustratos energéticos, algunos de los cuales son utilizados en forma inmediata tras la ingesta, mientras que otros son almacenados en el hígado, músculos y tejido adiposo para ser usados en otro momento. El glucógeno muscular y la glucosa pueden ser degradados por la vía aeróbica (oxidativa) o la anaeróbica lactacida (glucolítica), mientras que en ejercicios de resistencia la utilización de ácidos grasos se realiza a partir de la degradación de triglicéridos almacenados en el tejido celular subcutáneo. El metabolismo oxidativo mide la función de los sistemas cardiovascular y respiratorio. En cambio, el metabolismo anaeróbico solo involucra al tejido muscular.
\end{abstract}

Palabras claves: caballo, ejercicio, músculo, aeróbico, anaeróbico.

\section{ABSTRACT}

Three isoforms of MyHc have been characterized in the skeletal muscle of adult equines at the proteins level: types I, IIA and IIX. The distribution of these MyHCs defines three pure kinds of fibre which contain a single isoform (I+IIA and IIAX). The hybrid fibres IIAX exist in the locomotive muscles as a permanent and significant population. The adjusting response may take different forms depending on the nature of the stimulus: hypertrophy, reorganization (restructuring) without hypertrophy and a combined answer, when restructuring joins hypertrophy. The equines may take the most of different energetic subtrates, some of which are used immediately after the ingest, while others are stored in the liver, the muscles or adipose tissue in order to be used later. The muscular glycogen and the glucose may be degraded aerobically through the aerobic pathway or through the anaerobic pathway, while in the endurance exercises the use of fatty acids is accomplished from the degradation of fat stored in the subcutaneous cellular tissue. The oxidative metabolism measures the function of the cardiovascular and respiratory systems. On the contrary, the anaerobic metabolism involves only the muscular tissue.

\section{Adaptación muscular al entrenamiento}

El ejercicio induce cambios en la regulación y transcripción de genes específicos que inducen modificaciones en la cantidad de proteínas de una determinada isoforma en las fibras musculares.

Dependiendo de la naturaleza (tipo, frecuencia, intensidad y duración) y del estímulo (ejercicio o entrenamiento), la respuesta adaptativa puede tomar diferentes formas: 1) hipertrofia, cuando las fibras aumentan su tamaño pero mantienen su estructura basal, y sus propiedades fisiológicas y bioquímicas, 2) remodelación sin hipertrofia, cuando las miofibras no aumentan de tamaño pero sufren modificaciones de sus características estructurales y enzimáticas notorias, y que generalmente van acompañadas de cambios en la microvascularización, y 3) respuesta mixta, cuando se combina la remodelación con la hipertrofia. Yendo más lejos, la modalidad y amplitud de la respuesta depende significativamente del perfil muscular basal previo al entrenamiento.

Las modificaciones a nivel muscular se deben al incremento de la actividad contráctil que está asociada con los cambios inducidos por el entrenamiento hacia un músculo más oxidativo. Por lo tanto, las fibras de 
contracción rápida como así también los músculos con una alta proporción de las mismas, muestran una mayor adaptación al entrenamiento que las fibras de contracción lenta. Esta respuesta es más evidente en animales jóvenes e inactivos, ya que poseen una alta proporción de fibras glucolíticas de contracción rápida o tipo IIX, que los animales maduros y más activos con una proporción mayor de fibras oxidativas. Más allá de lo anteriormente expuesto, las adaptaciones musculares inducidas por el entrenamiento tienen implicancias fisiológicas que influyen en la generación de fuerza, velocidad y resistencia a la fatiga.

En general, la respuesta adaptativa del músculo esquelético al entrenamiento de larga duración y baja intensidad consiste en una mínima, si es que existe alguna, hipertrofia muscular. Sin embargo, una hipertrofia muscular específica puede ser estimulada con ejercicios de alta velocidad y corta duración y con ejercicios que generen un estiramiento máximo del sistema musculoesquelético, pero dentro del rango normal del mismo. Seis meses de entrenamiento convencional para salto induce una hipertrofia muscular selectiva de las fibras de tipo II. Otros estudios han reportado un aumento significativo y precoz (en menos de 3 meses) del diámetro de sección de las fibras de tipo I y IIA como consecuencia del entrenamiento (Rivero J.L.L. et al., 1995). Esto está parcialmente explicado por la conversión de fibras musculares en dirección IIX $\rightarrow$ IIA $\rightarrow$ I, ya que las fibras IIX y IIA muestran un diámetro de sección mayor que las fibras de tipo I. Por otra parte, otros estudios con caballos trotadores y Sangre Pura de Carrera (SPC), han mostrado cambios mínimos en la reducción del diámetro de sección de las fibras tipo II. Estas observaciones son difíciles de explicar cuando por otra parte se observa un prominente aumento de la masa muscular, fundamentalmente a nivel glúteo en caballos que se encuentran en entrenamiento. La única explicación para el incremento de la masa muscular sin un aumento del tamaño de las fibras musculares, consiste en un aumento en forma paralela de la cantidad de fibras (hiperplasia), como se ha observado en los humanos (Sjoström M. et al., 1991).

La distribución de las fibras musculares como así también de las cadenas de meromiosina pesada (MyHC) esta altamente influenciada por el entrenamiento. La buena performance en Enduro se correlaciona con un incremento en el porcentaje de fibras de tipo I y IIA y una reducción de las fibras de tipo IIX (Rivero J.L.L. et al., 1995) que va acompañada de la reducción de la MyHC-IIX y del aumento de la MyHC-IIA, mientras que la velocidad se correlaciona con una alta proporción de fibras de tipo II (Barrey B. et al., 1999). Por lo tanto, es posible diferenciar el potencial de un caballo para Enduro evaluando la composición del tipo de fibras en determinados músculos (Rivero J.L.L. \& Henckel P., 1996), aunque conclusiones diferentes se han obtenido en caballos SPC y trotadores. Además, varios otros estudios han mostrado un incremento en la isoforma híbrida I + IIA y en las fibras de tipo I. La transición de los tipos de fibras musculares durante el entrenamiento de resistencia se asemeja mucho a lo observado en el entrenamiento de Enduro. Este entrenamiento en caballos muestra un incremento en la relación de fibras IIA:IIX, y cuando el mismo es lo suficientemente prolongado hay un incremento en la relación de fibras de tipo I:II. Los entrenamientos de velocidad en caballos muestran un incremento del porcentaje de fibras de tipo IIA con el concomitante descenso de las fibras de tipo IIX y de su respectiva MyHC. En contraste con el entrenamiento de Enduro una disminución específica de las fibras de tipo I ha sido reportado en la fase inicial, y probablemente en forma transitoria con entrenamientos de alta intensidad.

Cuando varios estudios sobre el efecto del entrenamiento son combinados, es razonable pensar que los tipos de fibras musculares de transición sufren cambios en orden secuencial desde fibras de contracción rápida glucolíticas a otras más lentas y oxidativas (Ej. IIX $\rightarrow$ IIAX $\rightarrow$ IIA $\rightarrow$ IIA + I $\rightarrow$ I). La relación estímulo-respuesta entre la duración total del programa de entrenamiento y la magnitud de los cambios ocurridos han sido demostrados recientemente a nivel molecular (Rivero J.L.L. et al., 1995). Esta relación puede ser explicada en términos de umbral para la transición de fibras de tipo IIX $\rightarrow$ IIA durante la fase temprana de entrenamiento, y luego un nuevo umbral para la transición de fibras tipo IIA $\rightarrow$ I. De esta forma, una misma fibra es capaz de transformarse en forma completa desde fibra rápida a fibra lenta si posee un tiempo suficiente de entrenamiento. Es importante recordar que otras muchas isoformas proteicas como la sarcomérica, la proteína que regula la actina, y la proteína reguladora del calcio del retículo sarcoplásmico también se modifican. 
Tal vez la más precoz y común adaptación muscular al entrenamiento es el incremento de la actividad enzimática del metabolismo aeróbico, como las enzimas del ciclo de Krebs, de la cadena respiratoria mitocondrial y de la $\beta$ oxidación (Rivero J.L.L. et al., 1995). Estos cambios están asociados con el incremento de mitocondrias y de la densidad capilar. Las respuestas adaptativas más tardías involucran la mejora en la difusión de $\mathrm{O}_{2}$ y en la remoción de los desechos metabólicos (como el $\mathrm{CO}_{2}$ ).

La actividad de enzimas paso limitantes del metabolismo anaeróbico como la fosfofructoquinasa y lactato deshidrogenasa, no sufren variaciones 0 disminución durante el entrenamiento. Sin embargo, la velocidad de trote es altamente dependiente de la habilidad muscular para generar energía por la vía glucolítica (Ronéus N. \& Essén-Gustavsson B., 1997). Aunque el entrenamiento produce el aumento de la enzima AMP deaminasa y de otras enzimas involucradas en el ciclo de los nucleótidos de purinas como la creatin kinasa (CK), la concentración total de los mismos en el músculo no se ve afectado por el entrenamiento. El entrenamiento también ha mostrado un leve incremento en los depósitos de glucógeno almacenados en los músculos, que podría estar asociado con la reducción de enzimas glucolíticas, ya que la capacidad para movilizar glucógeno endógeno está parcialmente influenciada por la actividad de las enzimas anaeróbicas dentro de las fibras musculares. En caballos el entrenamiento de moderada intensidad incrementa los transportadores GLUT-4 en el músculo glúteo medio, pero éste incremento no está acompañado por un aumento en el trasporte de glucosa a través del sarcolema de la fibra muscular. Por otra parte, la captación de ácidos grasos libres desde el lecho vascular hacia el interior de la fibra, se ve aumentado como consecuencia del entrenamiento aeróbico por el incremento de la concentración de albúminas en el espacio extracelular (intersticio).

La consecuencia fisiológica más importante en respuesta al incremento de la masa muscular es la generación de un músculo más fuerte, ya que la generación de fuerza es proporcional al total del área de sección transversal de la masa de fibras reclutadas. A baja velocidad, esta adaptación tiene su efecto sobre el paso, causando una marcada reducción de la fase estática, como así también una marcada reducción en la duración total del mismo. Además, esta adaptación tiene un impacto importante sobre la performance en caballos de salto por intermedio del incremento en la generación de potencia de los miembros posteriores. Además, el incremento de fuerza permite una mejora en la aceleración y tal vez mejore la longitud del paso, haciendo que estas adaptaciones resulten de mucha utilidad en caballos que compiten en distancias cortas (Snow D.H. \& Valberg SJ., 1994). Sin embargo, es importante tener presente que el incremento de la potencia muscular va en detrimento del potencial aeróbico, ya que el aumento de la masa de fibras reclutadas y el incremento en la utilización del ATP ocurre en forma simultánea con el relativo desmejoramiento en la difusión del oxigeno hacia las fibras de mayor diámetro de sección (Essén-Gustavsson et al., 1989).

Desde el punto de vista fisiológico, la remodelación fenotípica de las miofibras con una mínima o nula hipertrofia en respuesta al entrenamiento, genera un músculo más resistente a la fatiga pero con una disminución intrínseca a la velocidad. El incremento de la resistencia a la fatiga se debe a un incremento en la capacidad oxidativa de cada miofibra. La reducción en la velocidad de contracción está asociada con la estimulación de determinados tipos de fibras musculares y con el aumento en la expresión de la isoforma lenta y otras isoformas de proteínas contráctiles. En una forma similar o recíproca a la descripta para los entrenamientos de resistencia, algunos programas de entrenamiento convencionales para potrillos SPC producen una disminución en el tamaño de las fibras de tipo II y una correspondiente disminución de la velocidad y de la fuerza de contracción (Valberg S.J., 1996). Es importante resaltar que debe buscarse el balance más apropiado (en tipos de fibras) para el tipo de disciplina hípica en la que compite el caballo. Los programas de entrenamiento para caballos SPC deben tener por finalidad el desarrollo de características musculares que optimicen el equilibrio entre velocidad, stamina (resistencia a la velocidad) y fuerza.

Los entrenamientos de resistencia consisten en ejercicios submáximos que mejoran el transporte y difusión del oxígeno, como así también el transporte de ácidos grasos insaturados que se metabolizan por la vía oxidativa con la consiguiente preservación de los 
depósitos de carbohidratos intracelulares. La disminución en la utilización del glucógeno intramuscular retarda la aparición de fatiga durante este tipo de ejercicios. Parece haber una alta probabilidad de que todas estas adaptaciones metabólicas sean las responsables del incremento en la resistencia en los individuos bien entrenados durante un periodo de mediano a largo plazo. Este incremento en la capacidad oxidativa de los músculos se correlaciona con el incremento en la captación máxima de oxígeno, y con una significativa reducción en la utilización del glucógeno como sustrato energético por la vía anaeróbica. Como consecuencia de esto, los caballos entrenados tienen un retraso en la acumulación de lactato y por consiguiente la acidosis aparece más tardíamente. Esto está acompañado por una mejora de la capacidad buffer y en una más eficiente excitaciónrelajación de las fibras musculares. En otras palabras el aumento de la resistencia (capacidad oxidativa) retarda la aparición de fatiga durante los ejercicios con un alto componente anaeróbico.

\section{Metabolismos energéticos y ejercicio}

Los equinos pueden aprovechar diferentes sustratos energéticos, algunos de los cuales son utilizados en forma inmediata tras la ingesta, mientras que otros son almacenados en el hígado, músculos y tejido adiposo para ser usados en otro momento. Finalmente, la energía presente en dichos sustratos es convertida a adenosina trifosfato (ATP) que es la forma en la que puede ser utilizada por el músculo.

La energía es obtenida mayoritariamente a partir de la degradación del fosfato de creatina, de los carbohidratos y de las grasas, que son sustratos que se encuentran tanto dentro como fuera del músculo. Estos sustratos energéticos pueden ser metabolizados por diferentes vías, ya que los depósitos intracelulares de ATP libre son muy escasos y luego de un corto periodo de tiempo se agotan.

El aporte energético durante el ejercicio no deriva de una única vía metabólica sino que, por el contrario, hay una integración del metabolismo aeróbico, anaeróbico, y de los sustratos energéticos metabolizados. El grado de integración metabólica va a depender del tipo de ejercicio al que esta sometido el animal. Los caballos que corren distancias no superiores a los 1.000 metros utilizan un $60 \%$ de energía producida anaerobicamente y un $40 \%$ producida en forma aeróbica, mientras que, a medida que la distancia aumenta (como en las carreras clásicas con distancias superiores a los 1.600 metros), aproximadamente el 80 $\%$ de la energía utilizada es generada por la vía aeróbica y solo un $20 \%$ proviene del metabolismo anaeróbico. Es decir, que cuanto mayor es la distancia de carrera mayor es el consumo de energía producida por la vía oxidativa, en detrimento de la producida por la vía glucolítica. Estos porcentajes indican que en ejercicios de máxima intensidad la fibra muscular puede tardar hasta un minuto en alcanzar la máxima producción de energía por la vía aeróbica. Para caballos que compiten en carreras de enduro, donde se corren distancias de hasta 160 kilómetros en un día a una velocidad que promedia generalmente los 16-18 Km./h, dependiendo de la superficie del terreno, la energía utilizada es generada aeróbicamente en un porcentaje superior al 94 $\%$. En otras palabras puede decirse que los ejercicios que no generan una frecuencia cardiaca superior a los 150 o 160 latidos por minuto, usan la vía aeróbica como generadora de energía. Esto se debe a que la vía oxidativa puede generar energía suficiente por unidad de tiempo como para satisfacer esa demanda energética de la fibra muscular.

En ejercicios de resistencia se utilizan como sustratos energéticos mayoritariamente el glucógeno, la glucosa y los ácidos grasos libres. La glucosa y los ácidos grasos libres se incrementan en sangre a los pocos minutos de haber comenzado el ejercicio, pero recién luego de haber degradado entre el 20 - 30\% del glucógeno almacenado en el músculo se activa la $\beta$ oxidación.

La intensidad de ejercicio a la cual la producción de energía por vía aeróbica se hace crítica varía. Esta variación se debe a varios factores, entre los que encontramos la disponibilidad de $\mathrm{O}_{2}$ que posee la fibra muscular, la concentración enzimática intracelular, la densidad mitocondrial, el nivel nutricional, si hubo o no precalentamiento previo y la velocidad a la que se corre la carrera.

La disponibilidad de $\mathrm{O}_{2}$ a nivel muscular en general siempre es suficiente, contrariamente a lo que se creía anteriormente. Por lo tanto, la activación del metabolismo anaeróbico se da cuando el metabolismo 
aeróbico esta funcionando al máximo de su capacidad y los requerimientos energéticos por unidad de tiempo siguen incrementándose. Por otra parte es importante tener en cuenta que dentro de un mismo músculo hay fibras musculares que, independientemente de la disponibilidad de $\mathrm{O}_{2}$, no pueden generar energía por la vía oxidativa debido a la ausencia de mitocondrias y de mioglobina, ambas vitales para el funcionamiento del metabolismo aeróbico.

\section{Bibliografia}

Barrey B., Valette JP., Jouglin M., et. al. (1999) Heritability of percentage of fast myosin heavy chains in skeletal muscles and relationship with performance. Equine Vet. J. Suppl., 30: 289-292.

Essén-Gustavsson B., McMiken D., Karlström K., et. al. (1989) Muscular adaptation of horses during intensive training and detraining. Equine Vet. J., 21: 27-33.
Rivero JLL., Ruz MC., Serrano A., et. al. (1995) Effects of a 3 months endurance training programme on skeletal muscle histochemistry in Andalusian, Arabian and Anglo-Arabian horses. Equine Vet. J., 27: 51-59.

Rivero JLL. and Henckel P. (1996) Muscle biopsy index for discriminating between endurance horses with different performance records. Res. Vet. Sci., 61: 49-54.

Ronéus N., Essén-Gustavsson B. (1997) Skeletal muscle characteristics and metabolic response to exercise in young Standardbreds. Am. J. Vet. Res., 58: 167-170.

Sjoström M., Lexell J., Eriksson A., et. al. (1991) Evidence of fibre hyperplasia in human skeletal muscles from healthy young men. Eur. J. Appl. Physiol., 62: 301-308.

Snow DH., Valberg SJ. (1994) Muscle anatomy, physiology and adaptations to exercise and training. In: Hodgson DR., Rose RJ. Eds. The athletic horse: principles and practice of equine sport medicine. Philadelphia: Saunders, 145-179.

Valberg SJ. (1996) Muscular causes of exercise intolerance in horses. Vet. Clin. North Am.: Equine Pract., 12: 495-515. 